\title{
SÍNDROME DA APNEIA E HIPOAPNEIA OBSTRUTIVA \\ DO SONO E O ENFOQUE FONOAUDIOLÓGICO: REVISÃO DE LITERATURA
}

\author{
Sleep obstructive apnea-hypoapnea syndrome \\ and the phonoaudiological focus: literature review
}

Priscila Goretti de Landa ${ }^{(1)}$, Heloisa Sawada Suzuki ${ }^{(2)}$

\begin{abstract}
RESUMO
Tema: Síndrome da Apneia e Hipoapneia Obstrutiva do Sono (SAHOS) é considerada um transtorno respiratório do sono, que afeta a população geral, com sintomas noturnos e diurnos que causam grande impacto na qualidade de vida do portador da síndrome. O interesse por tal patologia intensificou a partir do final dos anos de 1970 e recentemente vislumbra-se a fonoterapia como uma das alternativas para o tratamento da SAHOS. Objetivo: analisar as principais causas da síndrome da apneia e hipoapneia obstrutiva do sono e correlacioná-las ao trabalho fonoaudiológico. Conclusão: as causas mais encontradas relacionadas ao quadro da síndrome da apneia e hipoapneia obstrutiva do sono foram: redução da tonicidade do músculo genioglosso, comprimento excessivo de palato mole e úvula, obesidade, retrognatia e a prevalência em indivíduos do sexo masculino. O tratamento fonoaudiológico direcionado a estes indivíduos, seja ela em qual grau apresentar-se, proporciona uma melhora significativa do quadro, uma vez que o foco é a adequação da tonicidade e a mobilidade da musculatura orofacial.
\end{abstract}

DESCRITORES: Síndromes da Apneia do Sono; Ronco; Fonoterapia

\section{INTRODUÇÃO}

A Síndrome da Apneia e Hipoapneia Obstrutiva do Sono (SAHOS) é caracterizada, segundo a Academia Americana de Medicina do Sono, por episódios recorrentes de obstrução parcial ou total das vias aéreas superiores (VAS) e é definida como sendo uma doença crônica, progressiva, incapacitante, com alta mortalidade, no qual o fluxo de ar é diminuído na hipoapneia e completamente cessado na apneia ${ }^{1}$.

(1) Fonoaudióloga; Clínica Infantus, Centro de Apoio Solidaried'aids - Grupo CASA; Especializada em Motricidade Orofacial pelo CEFAC - Pós-Graduação em Saúde e Educação.

(2) Fonoaudióloga; Prefeitura Municipal de São Paulo, PMSP, São Paulo, SP; Santa Casa de Santo Amaro, São Paulo, SP; Docente do CEFAC - Pós-Graduação em Saúde e Educação; Doutora em Ciências pela Universidade Federal de São Paulo.

Conflito de interesses: inexistente
É considerado um transtorno respiratório do sono, que afeta a população geral, sendo mais frequente em pessoas de meia idade. A prevalência é de $4 \%$ nos homens e $2 \%$ nas mulheres. Devido aos recorrentes microdespertares, os indivíduos acometidos podem apresentar alterações funcionais, neurocognitivas e psicossociais. Alterações anatômicas também são sempre encontradas associadas a casos de SAHOS. Geralmente a geometria das VAS está alterada, podendo encontrar uma ou mais alterações ${ }^{2-5}$.

A redução da tonicidade dos músculos da faringe e genioglosso promovem o estreitamento das VAS, ocasionando um aumento da velocidade do fluxo aéreo, gerando uma vibração do palato mole e dos tecidos da faringe, produzindo, em última análise, o ronco, ruído que pode ocorrer durante o sono, gerado predominantemente na inspiração e causado pela vibração dos tecidos moles na orofaringe ${ }^{5}$.

O portador de SAHOS apresenta ronco interrompido pelos episódios de parada respiratória (ronco 
cíclico e intenso), enquanto os indivíduos que não possuem a síndrome apresentam ronco suave e contínuo (menos intenso) ${ }^{6,7}$.

A SAHOS é caracterizada por sintomas noturnos e diurnos. Nos sintomas noturnos, estão presentes as pausas respiratórias, sono agitado com múltiplos despertares, noctúria (devido aumento da secreção de fator natriurético atrial), sudorese, engasgos, insônia noturna (não é muito comum e está mais presente em mulheres). Dentre os diurnos estão: hipersonolência diurna, cefaleia matinal (podendo ser do tipo bilateral, frontal, occipital ou nucal e mais intensa quanto mais o paciente permanece dormindo, por conta do maior acúmulo de $\mathrm{CO}_{2}$ ), déficits neuro-cognitivos, déficits de memória e atenção (causado pela sonolência excessiva e fragmentação do sono REM), alterações de personalidade, redução da libido, sintomas depressivos (principalmente nas mulheres), ansiedade, hipertensão pulmonar, problemas sexuais, estresse oxidativo ${ }^{4,8-10}$.

Alguns fatores coadjuvantes ajudam no agravamento de casos de SAHOS, como a obesidade, hipertensão arterial, doenças cardiopulmonares como arritmias cardíacas, hipertensão pulmonar e insuficiência cardíaca, acidente vascular encefálico, álcool e acidentes de tráfego ${ }^{1,11}$.

O diagnóstico é obtido por meio de questionários médicos qualitativos e o exame de confirmação é realizado pela polissonografia assistida, considerada padrão ouro, e que é indispensável para a determinação da gravidade da doença. Três outros exames adjuvantes são utilizados para localização de sítios de obstrução, como a cefalometria, a nasofaringoscopia e a ressonância magnética ${ }^{4}$.

A Fonoaudiologia vem ampliando seu campo de atuação e investindo em estudos de novas patologias como a SAHOS. O interesse por tal patologia ganhou vulto a partir do final dos anos de 1970, após 30 anos de significantes estudos e descobertas que chamaram a atenção para a importância do sono e seus distúrbios, sobre a saúde.

Partindo dessa premissa, o objetivo do presente estudo é analisar os principais artigos relacionados à SAHOS e correlacioná-los ao trabalho fonoaudiológico.

\section{MÉTODOS}

Foi realizado um levantamento de 30 estudos que dizem respeito à SAHOS, para a realização de uma revisão bibliográfica, de escritos literários (como livros, artigos, periódicos de revistas e jornais, teses), de sites eletrônicos científicos e sob a forma de revisão de literatura, com preferência às publicações mais recentes (a partir de 1998).

\section{REVISÃO DA LITERATURA}

A transformação das VAS iniciou-se a duzentos e trezentos anos atrás, segundo muitos paleontologistas, que a consideram como a mais importante mudança anatômica da história dos ancestrais humanos. Essas mudanças incluem encurtamento da face, anteriorização do forame magno, redução do ângulo entre as partes horizontal e vertical da VAS e, principalmente, a descida da laringe, configurando o trato aéreo supralaríngeo mais longo e estreito, o que facilitou o desenvolvimento da fala e da linguagem. No entanto, essa nova anatomia possibilitou o surgimento da SAHOS por ter transformado a laringe em um segmento vulnerável, sem arcabouço e propensa ao colapso durante a inspiração, devido a maior relevância respiratória que esta estrutura recebeu ${ }^{12}$.

A SAHOS é considerada um problema de saúde pública. A prevalência varia com a faixa etária e com critérios utilizados para diagnosticar a síndrome. Dados epidemiológicos revelam que esse distúrbio apresenta alta incidência e prevalência, atingindo cerca de $4 \%$ da população feminina e $9 \%$ da masculina. Podendo acometer qualquer faixa etária, entretanto o pico de incidência está situado entre 30 e 60 anos ${ }^{2,9}$, com maior acometimento de homens obesos nesta faixa etária e mulheres antes da menopausa. É comum encontrar SAHOS entre pacientes que apresentam obesidade, acromegalia, hipertensão arterial, problemas cardíacos, diabetes e anormalidades craniofaciais $2,5,12,13$.

O efeito da idade na prevalência da SAHOS na população geral foi melhor estudado e avaliado em uma pesquisa com homens e mulheres, com idade compreendida entre 20 e 100 anos. Constatou-se que, em mulheres, a idade de maior prevalência foi acima de 65 anos, enquanto que nos homens o grupo etário está entre 45 e 65 anos. Mulheres em climatério apresentam maior prevalência de apneia do que as em pré-menopausa e quando mulheres estão submetidas à terapia de reposição hormonal pós-menopausa, segundo esta estatística, ainda apresentam menor incidência de quadros de apneia se comparado a homens. Esta pesquisa chamou atenção para o fato de que idosos apresentam quadro de maior gravidade que jovens ${ }^{14}$.

Existe uma correlação positiva entre idade, obesidade e circunferência do pescoço, ou seja, com o avançar da idade aumentam o peso e o tamanho da circunferência do pescoço (por acúmulo de gordura), que são fatores de risco para apneia. Estudos recentes mostram que a ação da musculatura dilatadora da faringe diminui com a idade, diminuindo o calibre das VAS, tanto em homens quanto em mulheres. Estes estudos alertam também para 
o tônus do músculo genioglosso, que é maior em mulheres, o que sugere um mecanismo de defesa e manutenção da permeabilidade das VAS e menor redução da luz da VAS, em posição decúbito dorsal, que a apresentada pelos homens ${ }^{12-14}$.

Na Figura 1 observa-se que dos $30(100 \%)$ artigos analisados, $18(60 \%)$ destes fazem referência à redução da tonicidade do músculo genioglosso levando ao estreitamento das VAS e promovendo o estreitamento das paredes laterais da faringe. Uma pesquisa realizada comprova que mudanças significativas na estrutura dos tecidos moles são responsáveis pelo estreitamento lateral das VAS nos pacientes apneicos durante o sono, e, comprovou que essa parede lateral é mais espessa em apneicos e roncadores devido a mudanças que ocorrem na configuração da via aérea, podendo predispor o sujeito apneico ao fechamento da mesma durante o sono. E outra pesquisa concluiu que o aumento da espessura da parede lateral da faringe, do comprimento do palato mole e do volume da língua são responsáveis pela diminuição do calibre da VAS e consequentemente pela apneia noturna ${ }^{6}$.

A oclusão das VAS durante o sono ocorre ao nível da faringe, principalmente orofaringe, por colapso decorrente do relaxamento das estruturas musculares que revestem o canal, em face da pressão negativa intraluminal inspiratória ${ }^{15,16}$. Cerca de 24 músculos participam das funções da faringe que incluem a deglutição, a fala e a respiração. Seis desses músculos tem como principal papel a permeabilidade da faringe e o mais estudado é o genioglosso $5,16,17$.

Durante o sono a atividade motora do conjunto fica diminuída devido à perda da tonicidade dos músculos das VAS levando ao colapso, que é produto final da interação entre fatores anatômicos e funcionais, que causam um desequilíbrio entre a pressão de sucção inspiratória e intrafaríngea e as forças dilatadoras dos músculos faríngeos das VAS, reduzindo sensivelmente a luz do canal e a passagem de ar. Com a falta de ventilação alveolar adequada há dessaturação de oxihemoglobina e, quando a parada respiratória é mais demorada, há aumento da pressão parcial de gás carbônico no sangue arterial ( $\mathrm{PaCO} 2)$ e com isso o centro respiratório é estimulado e a ventilação aumentada. Esse aumento da $\mathrm{PaCO} 2$, denominada hipercapnia, ativa os músculos dilatadores das VAS e diminui a colapsibilidade por aumentar a tensão das paredes das VAS. Em indivíduos apneicos essa resposta é diminuída e leva os indivíduos a microdespertares e a sensação de engasgos ${ }^{4-6,13}$.

A atividade dos músculos dilatadores da faringe aumenta à medida que a apneia progride, mas este aumento não é suficiente para estabelecer a permeabilidade da faringe. Este prejuízo muscular está presente somente no sono, o que condiz com o conceito de que a SAHOS é um distúrbio respiratório determinado pelo sono ${ }^{13,16}$.

Essa redução da tonicidade dos músculos da faringe e genioglosso acabam, em primeira instância, provocando o ronco, um ruído gerado predominantemente na inspiração e causado pela vibração dos tecidos moles da orofaringe. Envolvem o palato mole, a úvula, as tonsilas (amígdalas, pilares amigdalianos, base de língua, músculos e mucosa faríngea). Ao entrarem em relaxamento, mais especificamente no sono REM, os tecidos citados acima se tornam mais volumosos e flácidos, levando até à parada respiratória quando o ronco está associado a quadro de apneia ${ }^{14,18,19}$.

O ronco é um dos principais sintomas dos pacientes com SAHOS. Ele pode alcançar $85 \mathrm{~dB}$ e tende a ter a intensidade aumentada com o peso. É provocado por diversos fatores como flacidez de palato mole, úvula, língua e faringe (os tornam incapazes de manter a via aérea aberta durante a inspiração, sendo esta causa principal do ronco iniciado na fase adulta), comprimento excessivo de palato mole e úvula (produzem vibrações durante a respiração, obstruções respiratórias, como desvio de septo nasal e hipertrofia de língua, tonsilas palatinas e adenoides) ${ }^{4,14}$.

Conforme mostra a Figura 1, dos 30 textos analisados, 15 (50\%) deles fazem relação da apneia com o comprimento excessivo do palato mole e úvula, responsáveis pelo ronco. Este aumento acentuado do palato mole gera redução na nasofaringe e consequentemente maior contato entre o palato mole e a língua, contribuindo para o colapso nesta área. Pesquisa realizada com o auxilio de medidas cefalométricas, constataram que o comprimento do palato mole nos indivíduos apneicos é em torno de $12 \mathrm{~mm}$ maior que a dos indivíduos sadios ${ }^{3}$.

O aumento vertical do palato mole é resultado do prejuízo dos músculos palatinos nos pacientes apneicos. É possível que a atividade dos músculos palatinos tenha efeito no espessamento das paredes laterais da faringe, mudando a configuração da via aérea ${ }^{6}$.

O calibre das VAS também é afetado pela morfologia craniofacial. Por meio de exames cefalométricos é possível diagnosticar se o paciente apresenta a mandíbula deslocada mais posteriormente. De acordo com a Figura 1, dos $30(100 \%)$ estudos analisados, $15(50 \%)$ citam a retrognatia como uma das causas da apneia, e quanto maior este deslocamento, mais grave o quadro de apneia presente, porque este retroposiconamento mandibular é associado com o posicionamento posterior da base de língua ${ }^{2,5,14}$. 


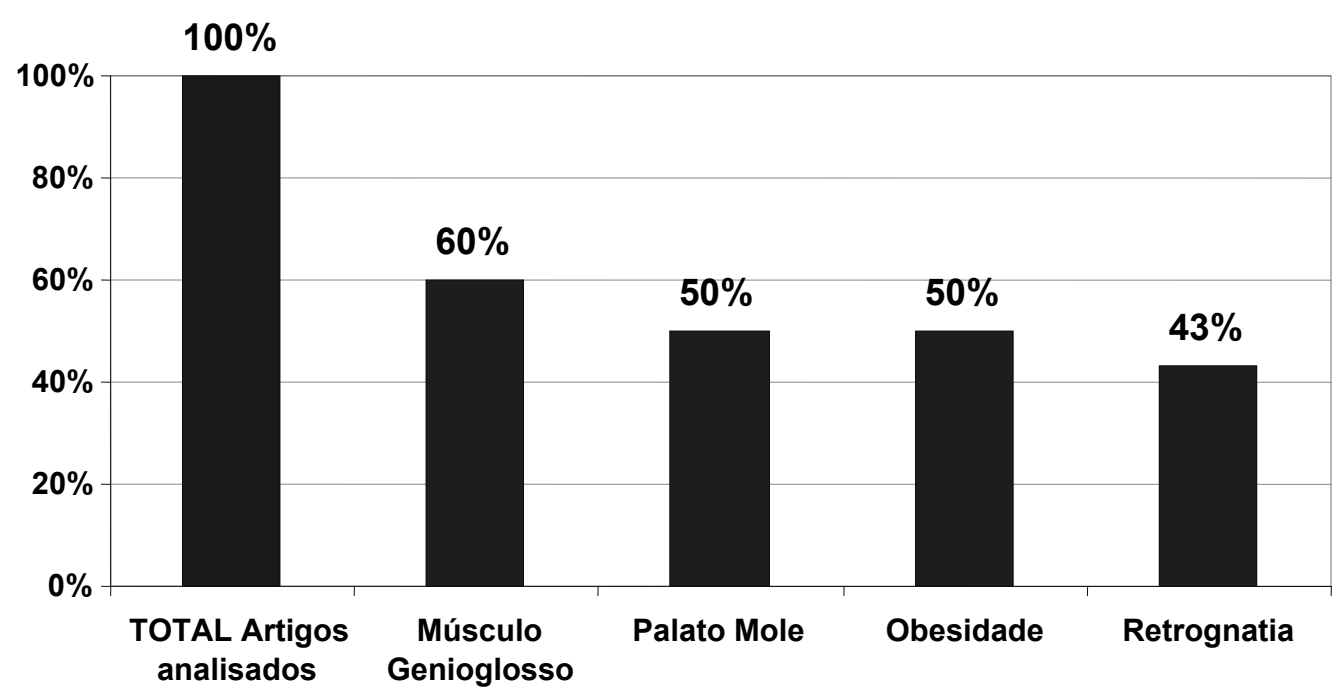

Figura 1 - Porcentagem de artigos que citam redução da tonicidade do músculo genioglosso, comprimento excessivo do palato mole e úvula, obesidade e retrognatia como fatores causais da Síndrome da Apneia e Hipoapneia Obstrutiva do Sono

Há uma relação estreita e recíproca entre os distúrbios respiratórios do sono e obesidade, sendo esta um importante fator patogênico da apneia. Dos $30(100 \%)$ artigos estudados, $15(50 \%)$ destes discorrem sobre a gravidade da SAHOS associada ao quadro de obesidade.

Aproximadamente $70 \%$ dos pacientes com SAHOS apresentam, pelo menos, sobrepeso. A obesidade é o único fator de risco de importância irreversível por causar modificações de tamanho e formato nas VAS, como o deposito de gordura parafaringeana, que acarreta em deformidade e estreitamento da orofaringe. Estas alterações só são possíveis de detectar em exames como a cefalometria e a ressonância nuclear magnética (RNM) 1,4,13,14.

$\mathrm{O}$ critério diagnóstico para SAHOS refere-se à presença de 5 ou mais episódios de apneia/hipoapneia (IAH) por hora de sono acompanhado de diminuição da saturação de oxigênio sanguíneo menos que $85 \%{ }^{2}$.

A classificação do nível de gravidade da SAHOS deve ser feito baseado nos índices polissonográficos, na intensidade dos sintomas, impacto nas funções cognitivas, sociais e profissionais em função do sexo, idade e profissão do indivíduo e nos resultados dos exames complementares ${ }^{2}$.

A síndrome é classificada em leve, quando acontecem de 5 a 15 eventos por hora; moderada, de 16 a 30 eventos por hora; e em severa, quando os eventos ultrapassam 30 por hora ${ }^{2}$.

Os exames realizados para detectar o quadro de SAHOS conforme a Figura 2, dos 30 (100\%) artigos pesquisados, $18(60 \%)$ referenciam a utilização do exame de polissonografia, nove $(30 \%)$ solicitam análise cefalométrica, nove (30\%) recorrem a ressonância magnética nuclear (RNM) e oito (27\%) realizam a nasofibrolaringoscopia para o diagnóstico e gravidade da apneia.

A polissonografia é um exame quantitativo especifico que permite a identificação e qualificação (número e duração) dos eventos respiratórios anormais. Consiste no monitoramento de parâmetros fisiológicos durante o sono para que seja diagnosticada a gravidade, o tipo de apnéia presente (central, obstrutiva ou mista), bem como alterações cardíacas, respiratórias e cerebrais. É considerado o exame de padrão ouro para o diagnóstico de SAHOS ${ }^{1,2,4,6,17,19-22}$.

A análise cefalométrica apresenta importante valor no diagnóstico etiológico da SAHOS e deve ser considerada entre os exames de rotina. Este exame corresponde a um procedimento diagnóstico necessário para se obter informações sobre anormalidades esqueléticas e em tecidos moles. Por meio da telerradiografia cefalométrica é possível obter medidas importantes como distância de base de língua e faringe, tamanho e posição de palato mole e úvula, tamanho do espaço aéreo faríngeo, volume e posição da língua, posição do osso hioide e relação dos planos mandibulares ${ }^{2,3,5,6,22}$.

A RNM tem sido muito usada para que seja evitada a radiação e para a obtenção de imagens em cortes axiais e sagitais da faringe, determinação de distribuição de gordura e de edemas, manifestado pelo aumento do volume de água nas circunjacências das VAS e obtenção do volume de sua luz $2,4,5,19,22$. 


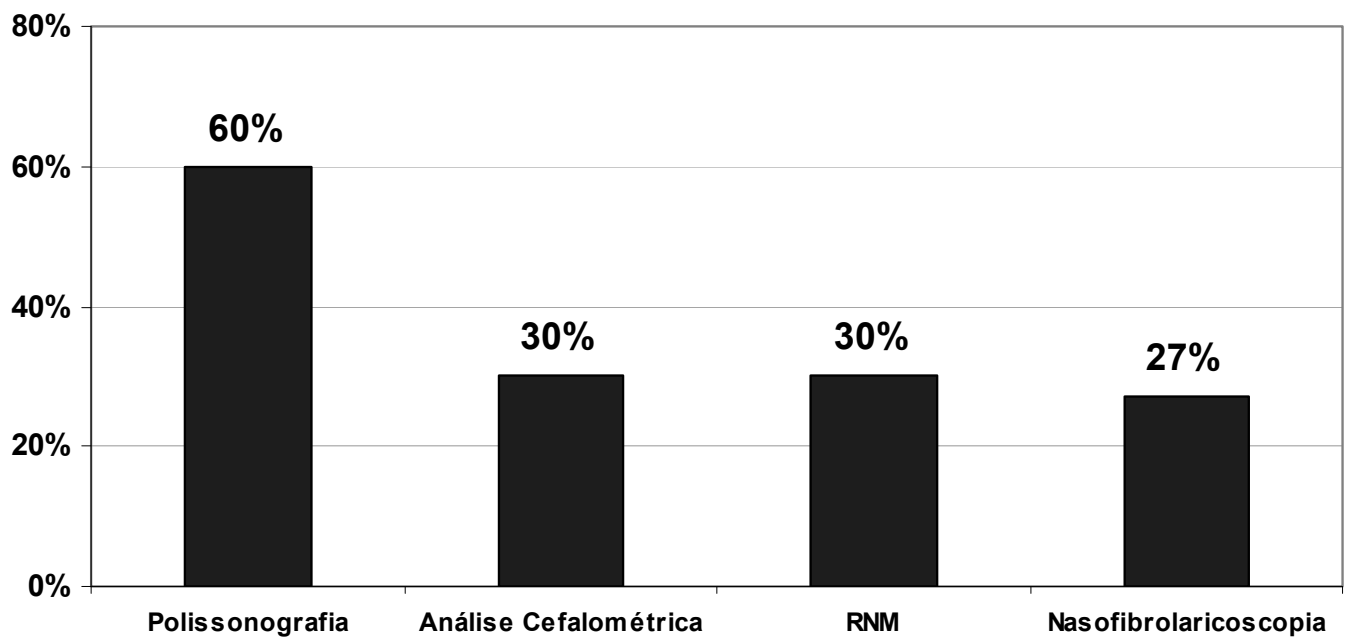

Figura 2 - Porcentagem dos artigos indicando os tipos de exames utilizados no diagnóstico da Síndrome da Apneia e Hipoapneia Obstrutiva do Sono

A nasofaringoscopia proporciona uma análise quase em tempo real das alterações das medidas de área de secção transversais da faringe durante a respiração, observando a função velofaríngea $2,5,6,19-22$.

Determinada a causa do problema, é traçado um ou mais tipos de tratamento. A Figura 3 ilustra os tratamentos utilizados hoje em dia para a SAHOS. Os tratamentos encontrados nas 30 literaturas pesquisadas são: comportamental, nove (30\%); cirúrgico, 14 (47\%); uso de aparelhos ortodônticos, nove (30\%); uso de pressão positiva de oxigênio (CPAP), 18 (60\%); e fonoterápico; citado por 12 (40\%).

O tratamento comportamental visa a redução e a eliminação de vícios. Incluem instruções para evitar o álcool, fumo, sedativos, anti-histamínicos, privação de sono, dormir em posição decúbito dorsal, refeições pesadas antes de dormir e aconselha os pacientes a exercitarem diariamente para desenvolver musculatura e perder peso ${ }^{1,2,4,7,17-23}$.

O tratamento cirúrgico objetiva a remoção de obstáculos mecânicos das VAS, remodelamento da região faringeana por meio de procedimentos sobre a base de língua, palato mole e paredes laterais da faringe, ou correção dos defeitos maxilo-mandibulares, visando avançar a mandíbula e/ou maxila para obter oclusão estável e aumentar o espaço posterior das VAS devido ao deslocamento ventral da musculatura da faringe, do genioglosso e do osso hioide. Essa última técnica cirúrgica, segundo a literatura pesquisada, pode trazer complicações e a mais comum é uma parestesia transitória do

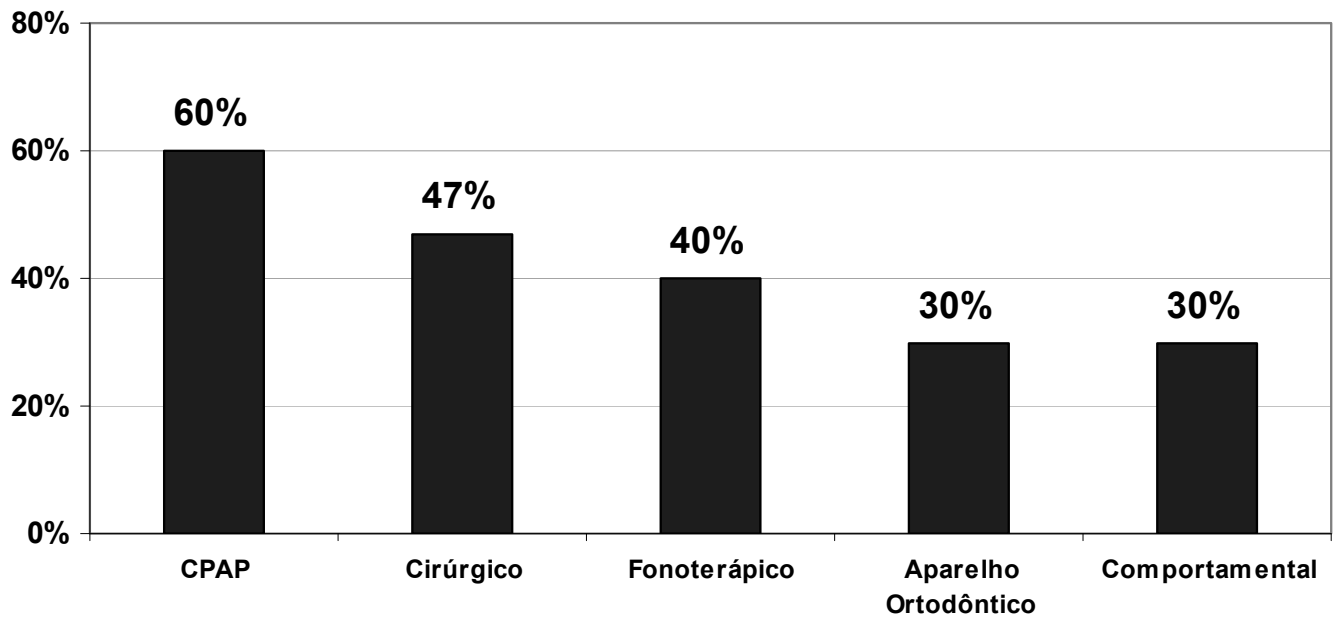

Figura 3 - Tratamentos encontrados e utilizados para a Síndrome da Apneia e Hipoapneia Obstrutiva do Sono 
nervo alveolar inferior 1,2,4,6,7,17,18,20-22,24-26. Ao que se refere o tratamento ortodôntico, os aparelhos intraorais (IAO) removíveis tem o objetivo de reposicionar anteriormente a língua e/ou a mandíbula prevenindo o colapso da faringe durante o sono, porém a indicação é preferencialmente para pacientes de SAHOS leve e quando a obstrução é ao nível da velofaringe 1,2,4-6,17-20,22,24-27.

O tratamento convencional e mais indicado até os dias de hoje é o aparelho de pressão positiva contínua nas vias aéreas - CPAP. É um método físico-mecânico de injeção de ar comprimido, utilizando uma máscara nasal que tem por princípio manter a desobstrução das vias aéreas durante o sono com a passagem contínua do ar. No entanto, é um tratamento que apresenta baixa adesão ao longo prazo por causar desconforto como ressecamento da mucosa nasal e oral, alguns pacientes sentem claustrofobia, e por ser um aparelho de custo elevado ${ }^{1,2,18-21}$.

Os tratamentos com CPAP e aparelho ortodôntico, citados anteriormente, podem atuar apenas de uma maneira paliativa, uma vez que não tratam efetivamente o fator que propiciou a instalação da síndrome. Na busca por tratamentos de baixo custo, maior aceitabilidade e resultados praticamente imediatos pelos indivíduos roncadores e apneicos, surgiu uma nova proposta de tratamento, baseada no trabalho oromiofuncional, realizada em consultório fonoaudiológico.

O tratamento fonoaudiológico, denominado por muitos autores como terapia miofuncional, começou a se desenvolver no Brasil, em 1996, na Santa Casa de Misericórdia em São Paulo, primeiramente aplicado a indivíduos roncadores. A terapia miofuncional constitui-se, inicialmente da conscientização do problema e da necessidade de sua correção, da melhora da postura corporal, da realização dos exercícios básicos e, por fim, de um período de reforço visando a manutenção dos novos padrões alcançados com a fonoterapia ${ }^{6,22}$.

A fonoterapia envolve primeiramente uma avaliação do sistema estomatognático, junto com a análise dos exames da nasofaringoscopia, análise cefalométrica, RNM, polissonografia e parecer ortodôntico. A seguir é traçada uma conduta terapêutica que visa em primeira instância exercícios de relaxamento cervical e dos órgãos fonoarticulatórios, exercícios respiratórios, exercícios específicos para adequar e estimular as funções do sistema estomatognático (principalmente a mastigação), exercícios orofaciais e articulatórios 18,19,22,24,28.

O trabalho fonoaudiológico nesta área ainda é incipiente, entretanto há relato de um estudo demonstrando bons resultados ${ }^{19}$.
A pesquisa envolvendo 20 indivíduos, sendo 15 homens e 5 mulheres submetidos a seis meses de terapia, apresentou um resultado de melhora de $50 \%$ do índice de apneia e hipoapneia (IAH) e ronco; em 12 homens e 3 mulheres e os outro 3 homens e as 2 mulheres obtiveram $40 \%$ de melhora do IAH e ronco. Dados consideráveis de diminuição do ronco, paradas respiratórias e sonolência diurna. Neste trabalho foram obtidos resultados quanto à mastigação, maior eficiência muscular de bucinador e lábios, adequação da deglutição, mímica facial, musculatura lingual e palatal ${ }^{19}$.

Em outro estudo, referente a um relato de caso de uma paciente de 60 anos de idade, com SAHOS de grau grave, que buscou tratamento mioterápico, observou-se após 12 sessões de fonoterapia, diminuição da tensão cervical, relaxamento da musculatura supra-hióidea, adequação do posicionamento do osso hióideo, rebaixamento do dorso da língua, normalidade da mobilidade do palato mole e adequação da mastigação. $\mathrm{O}$ exame polissonográfico acusou melhora do IAH de $44 /$ h para $3 / h$; desconsiderando um quadro de apneia grave para um distúrbio respiratório baixo, não mais caracterizado como SAHOS ${ }^{17}$.

Outra forma de tratamento dentro da terapia fonoaudiológica envolve também a reeducação da musculatura dilatadora da faringe no tratamento da roncopatia e da SAHOS ${ }^{29}$. Segundo publicações, há um aumento do tônus dessa musculatura que leva à diminuição ou eliminação dos roncos e da apneia do sono de todos os graus, mesmo em casos mais severos cuja indicação é CPAP (Continuous Positive Airway Pressure) ou BIPAP (Bilevel Positive Pressure Airway) (em casos de apneia central) 29,3

Dois casos de SAHOS grave, um feminino e um masculino, foram tratados com a terapia miofuncional oral, durante 16 semanas. O paciente do sexo feminino, 55 anos, apresentava queixa de roncos intensos, dificuldade respiratória durante o sono e lapsos de memória. A terapia voltou-se para padronização da postura e melhora da movimentação dos órgãos fonoarticulatórios. Foi constatada na polissonografia uma melhora de 40,4/h para 3,3/h IAH, com consequente diminuição dos microdespertares e melhora na saturação de oxigênio e na reavaliação fonoaudiológica melhora do tônus e da mobilidade das estruturas orofaciais e da coordenação fonoarticulatória, e segundo a paciente, melhora dos lapsos de memória. Já o paciente do sexo masculino, 37 anos, apresentava queixa de roncos, parada respiratórias, despertares frequentes, alteração de humor, dificuldade de concentração e déficit de memória. Após intervenção fonoaudiológica direcionada ao aumento do tônus 
muscular, melhora da mobilidade, relaxamento e coordenação dos órgãos fonoarticulatórios constatou-se, na polissonografia uma redução do IAH de $48,5 / \mathrm{h}$ para $8,6 / \mathrm{h}$, aumento na saturação de oxigênio e redução no índice de microdespertares. $\mathrm{Na}$ reavaliação miofuncional observou-se redução discreta da assimetria de bochechas, melhora do tônus e mobilidade de bochechas, língua, lábios, palato mole, bem como, da incoordenação fonoarticulatória e da respiração nasal ${ }^{22,24}$.

Estudo realizado no Hospital Universitário de Brasília abrangeu 9 pacientes, dentre eles, 3 roncadores sem SAHOS, 2 com SAHOS de grau leve, 2 com SAHOS de grau moderada e 2 com SAHOS de grau grave, com faixa etária entre 30 e 75 anos de idade. Os pacientes foram submetidos a um questionário de qualidade de vida, à Escala de Sonolência de Epworth (estabelece a propensão ao sono por meio de perguntas de situações cotidianas) e à Escala Subjetiva de Roncos, além da avaliação otorrinolaringológica e de todos os exames de precedência já citados para traçar a conduta terapêutica. $\mathrm{Na}$ avaliação fonoaudiológica de todos os pacientes analisados foram observadas as seguintes alterações: redução de tônus e de mobilidade de língua, bochechas, lábio e palato mole; qualidade vocal rouca e soprosa; estalos na articulação temporomandibular (ATM) à abertura de boca; respiração mista; mastigação com predomínio de um dos lados. Após intervenção fonoaudiológica foram observados: aumento da mobilidade e tônus da musculatura de língua, bochechas, lábios e palato mole em todos os pacientes. Redução da soprosidade e rouquidão vocal; cessação dos estaIos na ATM; melhora do padrão respiratório com predomínio do modo nasal e do padrão mastigatório, ressaltando que não houve intervenção direta na mastigação, na qualidade vocal e na ATM. Ao serem submetidos à polissonografia, estes pacientes apresentaram redução do IAH em todos os pacientes, principalmente nos SAHOS grave (de $48,5 / \mathrm{h}$ para $8,6 / \mathrm{h}$; e de $40,4 / \mathrm{h}$ para $3,3 / \mathrm{h}$ ), melhora na saturação do oxigênio e nos pacientes que só apresentavam queixa de ronco, houve uma redução expressiva no índice de microdespertares, nos roncos e na sonolência excessiva diurna ${ }^{22,24}$.

A terapia miofuncional direcionada aos indivíduos portadores de SAHOS, independente do grau, visa à adequação da postura, da sensibilidade, da propriocepção, do tônus e da mobilidade da musculatura orofacial e faríngea. As estruturas que devem ser priorizadas durante o trabalho são as que podem estar relacionadas com a obstrução pelo colapso das VAS durante o sono: assoalho da cavidade oral, lingual, especialmente os músculos genioglosso, mastigatórios (masseter, pterigóideo lateral e medial), bucinador, digástrico; palato mole e úvula; supra-hióideos e infra-hioideos; a musculatura faríngea, principalmente a dilatadora; e as funções de respiração, mastigação e deglutição ${ }^{15,16,19,22,24,28-30}$.

O trabalho fonoterápico junto a estes pacientes portadores de SAHOS consiste, portanto, na realização de exercícios isométricos, para aumento do tônus muscular, isotônicos, para melhora da mobilidade, e isocinéticos, indicados para o relaxamento e coordenação dos órgãos fonoarticulatórios e dos músculos envolvidos, citados anteriormente ${ }^{19,22,24,28}$.

Sabendo que as estruturas que envolvem o quadro de apneia obstrutiva do sono estão intimamente ligadas ao sistema respiratório e aos órgãos fonoarticulátorios, conclui-se com essa pesquisa que, sendo a fonoaudiologia responsável por tratar e proporcionar maior suporte a estas estruturas, a atuação da Fonoaudiologia junto à Medicina do Sono, torna-se, portanto, um elo importante para a recuperação e melhora da síndrome. Para tanto, mais estudos devem ser realizados, principalmente acompanhamento do estado do paciente antes e após a fonoterapia para a obtenção de dados mais consistentes a respeito dos efeitos do tratamento.

\section{CONCLUSÃO}

Diante do presente estudo as causas mais encontradas para apresentação da síndrome são: redução da tonicidade do músculo genioglosso, comprimento excessivo de palato mole e úvula, obesidade, retrognatia. Dentre estas causas, a atuação da fonoaudiologia, como comprovado e citado por $40 \%$ dos escritos literários analisados, é eficaz e se torna necessária para a adequação da tonicidade e da mobilidade da musculatura orofacial e faríngea, reduzindo assim o IAH e trazendo uma melhora global ao paciente. $O$ assunto é de grande importância, no entanto, poucas publicações científicas foram encontradas na área de fonoaudiologia. 


\begin{abstract}
Background: Sleep Obstructive Apnea-hypoapnea Syndromeis is considered a breath sleeping disorder that affects people in general, with its night and day symptoms which causes a great impact on life quality of the syndrome carrier. The concern on such pathology increased at the end of the 70's and nowadays spech therapy is seen as one of the possible treatments for SOAHS. Purpose: to analyze the main causes of sleep obstructive apnea-hypoapnea and to correlate them with the phonoaudiological work, since that this acts on the phonoarticulate organs, mainly rehabilitating the soft parts of the oropharynx musculature. Conclusion: the most recurrent causes related to sleep obstructive apnea-hypoapnea syndrome are: tonicity reduction of genioglossus muscle, too long soft palate and uvula, obesity, retruded, drug addiction and major prevalence in male sex are the most frequent causes related to Sleep Obstructive Sleep Apnea-Hypoapnea. The phonoaudiological treatment given to the patients, no matter what is the level of their problem, makes them feel better since that the focus is tonicity adequacy and orofacial musculature mobility.
\end{abstract}

KEYWORDS: Sleep Apnea Syndromes; Snoring; Speech Therapy

\section{REFERÊNCIAS}

1. Reimão R, Joo SH. Mortalidade da apneia obstrutiva do sono. Rev Assoc Med Bras. 2000; 46(1):52-6.

2. Ito FA, Ito RT, Moraes NM, Sakima T, Bezerra MLS, Meirelles RC. Condutas terapêuticas para tratamento da síndrome da apnéia e hipopnéia obstrutiva do sono (SAHOS) e da síndrome da resistência das vias aéreas superiores (SRVAS) com enfoque no aparelho anti-ronco (AAR-ITO). Rev Dent Press Ortodon Ortop Facial. 2005; 10(4):143-56.

3. Salles C, Campos PSF, Andrade NA, Daltro C. Síndrome da apneia e hipoapneia obstrutiva do sono: análise cefalométrica. Rev Bras Otorrinolaringol. 2005; 71(3):369-72.

4. Mancini MC, Aloe F, Tavares S. Apneia do sono em obesos. Arq Bras Endocrinol Metabol. 2000; 44(1):81-90.

5. Barbosa RC. Tratamento da síndrome da apneia obstrutiva do sono e do ronco por meio do aparelho reposicionador mandíbulo-lingual. Avaliação dos resultados por exame polissonográficos e de imagens obtidas por ressonância magnética. [tese] São Paulo (SP): Universidade de São Paulo; 1999. p.166.

6. Oliveira MRF, Souza TGG, Campiotto AR. Contribuição da fonoaudiologia no tratamento de indivíduos roncadores: relato de um caso. In: Marchesan IQ, Zorzi JL, Gomes ICD. Tópicos em fonoaudiologia 1997/1998. São Paulo: Lovise; 1998. p. 489-501.

7. Fairbanks DNF, Fairbanks DW. Ronco e apneia obstrutiva do sono In: Lopes Filho O, Campos CAH.
Tratado de otorrinolaringologia. São Paulo: Roca; 1995. p.199-213.

8. Stepanski EJ. The effect of sleep fragmentation on daytime function. Sleep. 2002; 25(3):268-76.

9. Shepertycky MR, Banno K, Kryger MH. Diferenças entre o homem e a mulher na apresentação clínica de doentes diagnosticados com síndrome de apneia obstrutiva do sono. Rev Portuguesa de Pneumol. 2006; 12(3):316-8.

10. Peppard PE, Young T, Palta M, Skatrud J. Prospective study of the association between sleepdisordered breathing and hypertension. $\mathrm{N}$ Engl J Med. 2000; 342(19):1378-84.

11. Yaggi HK, Concato J, Kernan WN, Lichtman JH, Brass LM, Mohsenin V. Obstructive sleep apnea as a risk factor for stroke and death. $\mathrm{N}$ Engl $\mathrm{J}$ Med. 2005; 353(19):2034-41.

12. Cahali MB. Consequências da síndrome da apneia obstrutiva do sono. Rev Bras Otorrinolaringol. 2007; 73(3):290.

13. Silva GA, Giacont LT. Síndrome das apneias/ hipopneias do sono (SAHOS). Simpósio: distúrbios respiratórios do sono. Ribeirão Preto. 2006; 39(2):185-94.

14. Martins AB, Tufik S, Moura SMGPT. Síndrome da apneia-hipopneia obstrutiva do sono. Fisiopatologia. J Bras Penumol. 2007; 33(1):93-100.

15. Séries F. Upper airway muscles awake and sleep. Sleep Med Rev. 2002; 6(3):229-42.

16. Ayappa I, Rapoport DM. The upper airway in sleep: physiology of the pharynx. Sleep Med Rev. 2003; 7(1):9-33.

17. Silva LMP, Aureliano FTS, Motta AR. Atuação fonoaudiológica na síndrome da apneia e hipoapneia obstrutiva do sono: relato de caso. Rev. 
CEFAC. 2007; 9(4): 490-6. dx.doi.org/10.1590/ S1516-18462007000400009.

18. Bento PB. Propostas fonoaudiológicas ao paciente roncador. [monografia] Londrina (PR): CEFAC - Pós-Graduação em Saúde e Educação; 2000.

19. Guimarães K. Alterações no tecido mole de orofaringe em portadores de apneia obstrutiva do sono. J Bras Fonoaudiol. 1999; 1:69-75.

20. - Burger RCP, Caixeta EC, Di Ninno CQMS. A relação entre apneia do sono, ronco e respirador oral. Rev. CEFAC. 2004; 6(3):266-71.

21. Amaral AK, Carvalho MM, Silva HJ. Respiração oral e suas relações com o ronco noturno e a síndrome da apneia obstrutiva do sono. J Bras Fonoaudiol. 2004; 5(18):60-2.

22. Pitta DBS. Avaliação da eficácia da intervenção fonoaudiológica - terapia miofuncional - em pacientes roncadores com e sem apneia obstrutiva do sono. [dissertação] Brasília (DF): Universidade de Brasília; 2005. p.120.

23. Almeida $M A O$, Teixeira $A O B$, Vieira LS, Quintão CCA. Tratamento da síndrome da apneia e hipoapneia obstrutiva do sono com aparelhos intrabucais. Rev Bras Otorrinolaringol. 2006; 72(5):699-703.

24. Martinho FL, Zonato Al, Bittencourt LRA, Gregório CL, Gregório LC, Tufik S. Indicação cirúrgica otorrinolaringológica em um ambulatório para pacientes com síndrome da apneia e hipoapneia obstrutiva do sono. Rev Bras Otorrinolaringol. 2004; 70(1):46-51.
25. Neto LM, Fava AS, Lopes HC, Stamm A. Estudo epidemiológico das alterações da cavidade nasal associada à síndrome da apneia e hipoapneia obstrutiva do sono (SAHOS). Rev Bras Otorrinologaringol. 2005; 71(4):464-6.

26. Pitta DBS, Pessoa AF, Sampaio ALL, Rodrigues $\mathrm{RN}$, Tavares MG, Tavares P. Terapia miofuncional oral aplicada a dois casos de síndrome da apneia obstrutiva do sono grave. Arq Int Otorrinolaringol. 2007; 11(3):452-4.

27. Quintela MM. Para onde caminha a terapia com aparelhos orais na solução do ronco e da apnéia obstrutiva do sono? Pesquisas, protocolos e perspectivas. In: Reimão R. Sono. Medicina do sono: neurociências, evoluções e desafios. São Paulo: APM; 2007. p. 69-79.

28. Marquesan IQ. Motricidade oral: visão clinica do trabalho fonoaudiológico integrado com outras especialidades. 2. ed. São Paulo: Pancast; 1999. p.24-81.

29. Bertolini MM. Reeducação da musculatura dilatadora da faringe no tratamento multidisciplinar da roncopatia e da síndrome da apneia obstrutiva do sono. In: Reimão R. Sono: sono normal e doenças do sono. São Paulo: APM; 2004. p. 65-9.

30. Bertolini MM. Roncopatia e SAOS: tratamento fonoaudiológico reabilitação da musculatura dilatadora da faringe. In: Reimão R. Sono. Medicina do sono: neurociências, evoluções e desafios. São Paulo: APM; 2007. p. 86-8.

RECEBIDO EM: 25/08/2008

ACEITO EM: 18/05/2009

Endereço para correspondência:

Priscila Goretti de Landa

Av Barão do Rio Branco, 4447

Juiz de Fora - MG

CEP: 36026-500

E-mail: pridelanda@ hotmail.com 